\title{
Diffraction theory of nanotwin superlattices with low symmetry phase
}

\author{
Yu U. Wang* \\ Department of Materials Science and Engineering, Virginia Tech, Blacksburg, Virginia 24061, USA \\ (Received 6 June 2006; revised manuscript received 7 August 2006; published 18 September 2006)
}

\begin{abstract}
A nanotwin diffraction theory is developed. It predicts an adaptive diffraction phenomenon, where the Bragg reflection peaks are determined by coherent superposition of scattered waves from individual twin-related nanocrystals and adaptively shift along the twin peak splitting vectors in response to a change in the twin variant volume fraction. Application of this theory to tetragonal phase explains the intrinsic lattice parameter relationships of monoclinic $M_{C}$ phase recently discovered in ferroelectric $\mathrm{Pb}\left[\left(\mathrm{Mg}_{1 / 3} \mathrm{Nb}_{2 / 3}\right)_{1-x} \mathrm{Ti}_{x}\right] \mathrm{O}_{3}$ and $\mathrm{Pb}\left[\left(\mathrm{Zn}_{1 / 3} \mathrm{Nb}_{2 / 3}\right)_{1-x} \mathrm{Ti}_{x}\right] \mathrm{O}_{3}$.
\end{abstract}

DOI: 10.1103/PhysRevB.74.104109

PACS number(s): 61.10.Dp, 61.12.Bt, 61.14.Dc

Ferroelastic crystals usually consist of structural twins to accommodate the spontaneous lattice distortion and minimize the elastic strain energy. ${ }^{1}$ The twin-related domains are crystallographically equivalent structural variants of different orientations of the same low-symmetry phase (e.g., tetragonal, rhombohedral, etc). They often self-assemble into highly regular (or "ordered") patterns. ${ }^{2}$ When the twin boundary energy density is small, the twin microstructure can miniaturize to form nanotwin superlattice, ${ }^{3}$ as occurred in martensitic crystals. ${ }^{4}$ Since the nanodomain size is much smaller than the coherence length of diffraction radiation, scattered waves from individual nanodomains coherently superimpose in diffraction. Therefore, the relative phase angles of the scattered waves must be considered. The purpose of this paper is to develop a nanotwin diffraction theory and report an adaptive Bragg reflection phenomenon, which explains the experimental observations in martensitic crystals ${ }^{4}$ and more recently in ferroelectric $\mathrm{Pb}\left[\left(\mathrm{Mg}_{1 / 3} \mathrm{Nb}_{2 / 3}\right)_{1-x} \mathrm{Ti}_{x}\right] \mathrm{O}_{3}$ and $\mathrm{Pb}\left[\left(\mathrm{Zn}_{1 / 3} \mathrm{Nb}_{2 / 3}\right)_{1-x} \mathrm{Ti}_{x}\right] \mathrm{O}_{3} \cdot{ }^{5-8}$ Similar adaptive diffraction phenomena should also occur in other nanoscale superlattices of low symmetry phases.

Nanotwins are believed to form in tetragonal ferroelectric $\mathrm{Pb}\left[\left(\mathrm{Mg}_{1 / 3} \mathrm{Nb}_{2 / 3}\right)_{1-x} \mathrm{Ti}_{x}\right] \mathrm{O}_{3}$ and $\mathrm{Pb}\left[\left(\mathrm{Zn}_{1 / 3} \mathrm{Nb}_{2 / 3}\right)_{1-x} \mathrm{Ti}_{x}\right] \mathrm{O}_{3},{ }^{9,10}$ where a new intermediate monoclinic $M_{C}$ phase ${ }^{5-8}$ is found to be related to the conventional tetragonal phase through three intrinsic lattice parameter relationships. ${ }^{10}$ The tetragonal nanotwin hypothesis explains these intrinsic relationships based on a nanodomain averaging effect in low-resolution diffraction used to measure the lattice parameters, ${ }^{9,10}$ because significant reflection peak broadening is expected for diffractions of individual nanodomains. However, single-crystal mesh scans of high-resolution x-ray and neutron diffractions well resolve a characteristic fine triplet peak structure around the (200) Bragg reflection spot, which is assumed to consist of (200) twin peaks from two $a$ domains and a (020) single peak from one $b$ domain of the monoclinic $M_{C}$ phase. $^{5-8}$ Therefore, if the observed monoclinic $M_{C}$ phase is indeed tetragonal nanotwins, this diffraction phenomenon needs a rigorous theoretical explanation. Application of this developed adaptive diffraction theory to tetragonal nanotwins solves this issue.

In this paper, we consider a one-dimensional nanotwin superlattice with a bilayer structural basis, since the size of each twin variant domain along the layer plane is much greater than the nanoscale layer thickness. The structure factor of the nanotwin superlattice can be written as

$$
\tilde{n}_{\text {basis }}(\mathbf{k})=\widetilde{n}_{1}(\mathbf{k}) * \tilde{\theta}_{1}(\mathbf{k})+\widetilde{n}_{2}(\mathbf{k}) * \tilde{\theta}_{2}(\mathbf{k}),
$$

where a tilde $\sim$ above a function indicates its Fourier transform, an asterisk $*$ represents convolution operation, $n_{1}(\mathbf{r})$ and $n_{2}(\mathbf{r})$ are the electron density distributions in an ideal (infinite) crystal with the same crystal structure and orientation of respective twin layers of the bilayer basis, and $\theta_{1}(\mathbf{r})$ and $\theta_{2}(\mathbf{r})$ are the shape functions describing twin layers of the bilayer basis, which assumes one inside each respective layer and zero outside. The function $n_{\text {basis }}(\mathbf{r})=n_{1}(\mathbf{r}) \theta_{1}(\mathbf{r})+n_{2}(\mathbf{r}) \theta_{2}(\mathbf{r})$ describes the electron density distribution in the bilayer basis. Using the structure factors $\widetilde{n}_{1}^{0}(\mathbf{k})$ and $\widetilde{n}_{2}^{0}(\mathbf{k})$ of respective twin layer crystals, where $n_{1}^{0}(\mathbf{r})$ and $n_{2}^{0}(\mathbf{r})$ describe the electron density distribution in the basis of respective twin layer crystals, the Fourier transform $\widetilde{n}_{1}(\mathbf{k})$ and $\widetilde{n}_{2}(\mathbf{k})$ become

$$
\begin{aligned}
& \widetilde{n}_{1}(\mathbf{k})=\sum_{\mathbf{K}^{(1)}} V^{-1} \widetilde{n}_{1}^{0}(\mathbf{k}) \delta\left(\mathbf{k}-\mathbf{K}^{(1)}\right), \\
& \widetilde{n}_{2}(\mathbf{k})=\sum_{\mathbf{K}^{(2)}} V^{-1} \widetilde{n}_{2}^{0}(\mathbf{k}) \delta\left(\mathbf{k}-\mathbf{K}^{(2)}\right),
\end{aligned}
$$

where $V$ is the primitive cell volume of the crystal, $\delta(\mathbf{k})$ is Dirac delta function in three-dimensional reciprocal space, and $\mathbf{K}^{(1)}$ and $\mathbf{K}^{(2)}$ collectively represent all reciprocal lattice sites of respective twin layer crystals.

The nanotwin superlattice is formed by periodically repeating the bilayer basis through a specific translation vector $\mathbf{L}$, which is the primitive lattice translation of the onedimensional nanotwin superlattice. The electron density distribution in the superlattice is $n(\mathbf{r})=\sum_{s} n_{\text {basis }}(\mathbf{r}-s \mathbf{L})$, where the summation is over integer $s$ running from $-\infty$ to $\infty$, i.e., assuming that the total thickness of nanotwin superlattice is much greater than the thickness of its bilayer basis. The Fourier transform of function $n(\mathbf{r})$ is

$$
\widetilde{n}(\mathbf{k})=\widetilde{n}_{\text {basis }}(\mathbf{k}) \sum_{s} e^{-i s \mathbf{k} \cdot \mathbf{L}} .
$$

Substituting Eqs. (1) and (2) into Eq. (3) yields 


$$
\begin{aligned}
\widetilde{n}(\mathbf{k})= & V^{-1}\left[\sum_{\mathbf{K}^{(1)}} \widetilde{n}_{1}^{0}\left(\mathbf{K}^{(1)}\right) \tilde{\theta}_{1}\left(\mathbf{k}-\mathbf{K}^{(1)}\right)\right. \\
& \left.+\sum_{\mathbf{K}^{(2)}} \widetilde{n}_{2}^{0}\left(\mathbf{K}^{(2)}\right) \tilde{\theta}_{2}\left(\mathbf{k}-\mathbf{K}^{(2)}\right)\right] \sum_{s} e^{-i s \mathbf{k} \cdot \mathbf{L}} .
\end{aligned}
$$

The crystal lattices and reciprocal lattices of twin layer crystals are uniquely related by the twinning deformation matrix $\hat{\mathbf{A}}:{ }^{1,11} \mathbf{r}^{(2)}=\hat{\mathbf{A}} \mathbf{r}^{(1)}, \mathbf{K}^{(2)}=\hat{\mathbf{A}}^{-T} \mathbf{K}^{(1)}$, where $\mathbf{r}^{(1)}$ and $\mathbf{r}^{(2)}$ are lattice site position vectors before and after deformation, respectively, $\mathbf{K}^{(1)}$ and $\mathbf{K}^{(2)}$ denote the corresponding twin pairs of reciprocal lattice sites (i.e., Bragg reflection peak splitting) due to twinning deformation, and the symbol $-T$ represents matrix inverse transpose operation. The matrix $\hat{\mathbf{A}}$ is a special case of the invariant plane deformation matrix and has the following form: ${ }^{1,11}$

$$
\hat{\mathbf{A}}=\hat{\mathbf{I}}+\gamma \mathbf{S} \otimes \boldsymbol{\tau}, \quad \hat{\mathbf{A}}^{-T}=\hat{\mathbf{I}}-\gamma \boldsymbol{\tau} \otimes \mathbf{S},
$$

where $\hat{\mathbf{I}}$ is identity matrix, $\gamma$ is the magnitude of twinning shear strain, the symbol $\otimes$ represents vector dyadic product, $\boldsymbol{\tau}$ is a unit vector normal to the twin plane, and $\mathbf{s}$ is a unit vector along the twinning shear direction $(\boldsymbol{\tau}$ and $\mathbf{s}$ are perpendicular to each other, and twinning deformation does not change unit cell volume). The Bragg reflection peak splitting is characterized by the vector difference between the corresponding twin pair of fundamental sites $\mathbf{K}^{(1)}$ and $\mathbf{K}^{(2)}$,

$$
\Delta \mathbf{K}^{(1)}=\mathbf{K}^{(2)}-\mathbf{K}^{(1)}=-\left(\gamma \mathbf{s} \cdot \mathbf{K}^{(1)}\right) \boldsymbol{\tau},
$$

which shows that the peak splits along the twin plane normal direction.

For convenience, we select twin plane normal direction as $x$ axis and twinning shear direction as $z$ axis to setup our rectangular Cartesian coordinate system. Thus, $\boldsymbol{\tau}=(1,0,0)$, $\mathbf{s}=(0,0,1)$, and $K_{x}^{(2)}=K_{x}^{(1)}-\gamma K_{z}^{(1)}, K_{y}^{(2)}=K_{y}^{(1)}, K_{z}^{(2)}=K_{z}^{(1)}$. The shape functions $\theta_{1}(\mathbf{r})$ and $\theta_{2}(\mathbf{r})$ are window function of height 1 in region $x \in\left[-T_{1}, 0\right]$ and $x \in\left[0, T_{2}\right]$, respectively, where $T_{1}$ and $T_{2}$ are the thicknesses of respective twin layers of the bilayer basis, and $T=T_{1}+T_{2}=\mathbf{L} \cdot \boldsymbol{\tau}$ is the bilayer basis thickness. The Fourier transform $\widetilde{\theta}_{1}(\mathbf{k})$ and $\tilde{\theta}_{2}(\mathbf{k})$ are

$$
\tilde{\theta}_{1}(\mathbf{k})=f_{1}\left(k_{x}\right) \delta\left(k_{y}\right) \delta\left(k_{z}\right), \quad \tilde{\theta}_{2}(\mathbf{k})=f_{2}\left(k_{x}\right) \delta\left(k_{y}\right) \delta\left(k_{z}\right),
$$

where $\delta(\kappa)$ is a one-dimensional Dirac delta function, and

$$
f_{1}(\kappa)=\frac{2}{\kappa} \sin \frac{\kappa T_{1}}{2} e^{i\left(\kappa T_{1} / 2\right)}, \quad f_{2}(\kappa)=\frac{2}{\kappa} \sin \frac{\kappa T_{2}}{2} e^{-i\left(\kappa T_{2} / 2\right)},
$$

which describe the broadening of fundamental peaks $\mathbf{K}^{(1)}$ and $\mathbf{K}^{(2)}$, respectively, due to nanoscale layer thickness and their phase angles. The peak broadening is in the twin plane normal direction along the twin peak splitting vector $\Delta \mathbf{K}^{(1)}$. Substituting Eq. (7) into Eq. (4) yields

$$
\begin{aligned}
\tilde{n}(\mathbf{k})= & \frac{1}{V T} \sum_{\mathbf{K}^{(1)}} \sum_{\kappa^{(s)}} g\left(\kappa^{(s)}, \mathbf{K}^{(1)}\right) \delta\left(k_{x}-K_{x}^{(1)}-\kappa^{(s)}\right) \\
& \times \delta\left(k_{y}-K_{y}^{(1)}\right) \delta\left(k_{z}-K_{z}^{(1)}\right),
\end{aligned}
$$

where

$$
\begin{aligned}
g\left(\kappa^{(s)}, \mathbf{K}^{(1)}\right)= & \widetilde{n}_{1}^{0}\left(\mathbf{K}^{(1)}\right) f_{1}\left(\kappa^{(s)}\right)+\widetilde{n}_{2}^{0}\left(\mathbf{K}^{(1)}+\Delta \mathbf{K}^{(1)}\right) \\
& \times f_{2}\left(\kappa^{(s)}+\gamma \mathbf{s} \cdot \mathbf{K}^{(1)}\right),
\end{aligned}
$$

and $\kappa^{(s)}$ collectively represents the solutions of the following equation for all possible integer values $s$,

$$
\kappa^{(s)}=\frac{2 \pi s-\mathbf{L} \cdot \mathbf{K}^{(1)}}{\mathbf{L} \cdot \boldsymbol{\tau}} .
$$

Equation (9) shows that the Bragg reflection peaks of nanotwin superlattice are located at $\mathbf{k}^{(s)}=\left(K_{x}^{(1)}+\kappa^{(s)}, K_{y}^{(1)}, K_{z}^{(1)}\right)$ in reciprocal space, which are distributed along the lines normal to twin plane and passing both $\mathbf{K}^{(1)}$ and $\mathbf{K}^{(2)}$. In fact, $\mathbf{K}^{(s)}$ are the reciprocal superlattice sites of the nanotwins, whose reflection intensities are determined by the function $g\left(\kappa^{(s)}, \mathbf{K}^{(1)}\right)$ given in Eq. (10). Note that the superlattice site distribution, twin peak splitting, and peak broadening are all along the same lines normal to the twin plane.

It is worth noting that for nanotwin superlattices of low symmetry phases, the superlattice sites $\mathbf{k}^{(s)}$ generally do not coincide with the fundamental reflection sites $\mathbf{K}^{(1)}$ and $\mathbf{K}^{(2)}$ of the twin crystals, i.e., the values $\boldsymbol{\kappa}^{(s)}$ given in Eq. (11) do not reduce to 0 or $-\boldsymbol{\gamma} \mathbf{s} \cdot \mathbf{K}^{(1)}$. Therefore, the fundamental reflection peaks of the twin crystals at $\mathbf{K}^{(1)}$ and $\mathbf{K}^{(2)}$ will disappear, and new reflection peaks will appear at the reciprocal superlattice sites $\mathbf{k}^{(s)}$. This feature leads to an adaptive diffraction phenomenon, where the Bragg reflection peaks are determined by coherent superposition of scattered waves from individual twin-related nanodomains and, as will be shown later, these peaks adaptively move along the twin peak splitting vectors $\Delta \mathbf{K}^{(1)}$ in response to a change in the twin variant volume fraction. As a result, the lattice parameters measured from $\mathbf{k}^{(s)}$ are different from those of the constituent crystals instead are a specific combination of them.

It is also noteworthy that only a few superlattice peaks $\mathbf{k}^{(s)}$ in the immediate vicinity of the fundamental sites $\mathbf{K}^{(1)}$ and $\mathbf{K}^{(2)}$ could possibly have visible intensities. According to Eq. (8), the peak broadening in $\Delta \mathbf{K}^{(1)}$ direction has a typical full-width broadness of $4 \pi / T_{1}$ and $4 \pi / T_{2}$ for $\mathbf{K}^{(1)}$ and $\mathbf{K}^{(2)}$, respectively. As follows from Eq. (11), the neighboring superlattice sites are spaced by a distance $2 \pi / T$ in $\Delta \mathbf{K}^{(1)}$ direction. Therefore, approximately $2 T / T_{1}$ and $2 T / T_{2}$ superlattice sites around each respective $\mathbf{K}^{(1)}$ and $\mathbf{K}^{(2)}$ could produce visible reflection peaks, depending on the magnitudes and relative phase angle of the two terms in Eq. (10). As shown later for $(H 00)$ reflection of tetragonal nanotwins, when $H m \gamma<2$, where $m$ is the number of (101) atomic layers in bilayer basis, a single strong superlattice peak appears between the twin pair $\mathbf{K}^{(1)}$ and $\mathbf{K}^{(2)}$ and adaptively shifts along $\Delta \mathbf{K}^{(1)}$ determined by the twin variant volume fraction. When $m$ becomes significantly larger, less broadened peaks concentrate on individual $\mathbf{K}^{(1)}$ and $\mathbf{K}^{(2)}$ and consequently lose overlapping, leading to a transition to a conventional diffraction 

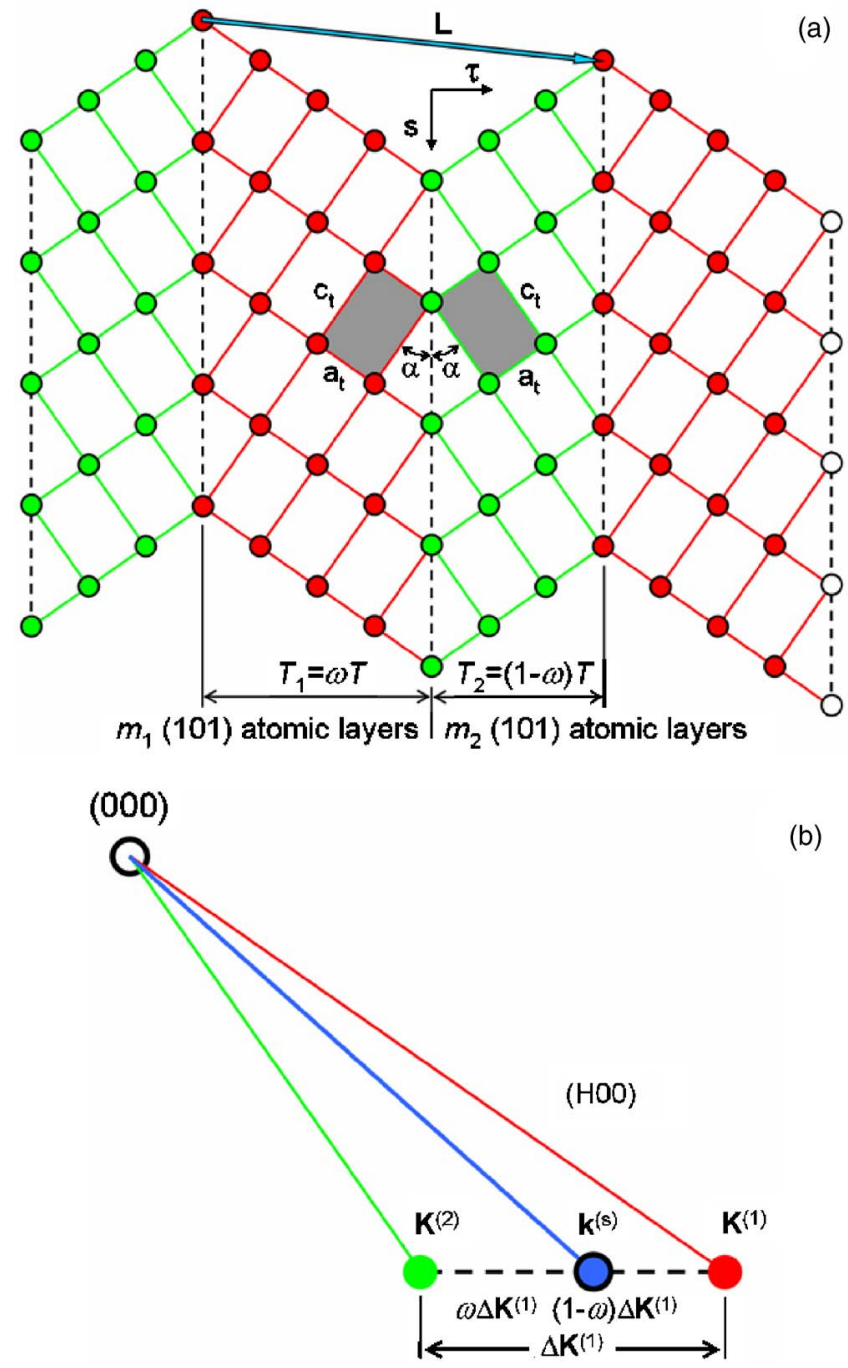

FIG. 1. (Color online) (a) Schematic of tetragonal nanotwin superlattice. (101) twin planes are indicated by dashed lines. A tetragonal unit cell is highlighted by gray shadow in respective twin variants. The primitive superlattice translation vector is $\mathbf{L}$. The volume fractions of the first and second twin variants are $\omega$ and $1-\omega$, respectively. The bilayer basis thickness is $T$. (b) Adaptive diffraction phenomenon, where the Bragg reflection peak $\mathbf{k}^{(s)}$ moves between the vanished fundamental peaks $\mathbf{K}^{(1)}$ and $\mathbf{K}^{(2)}$ of twin-related nanodomains in response to a change in $\omega$.

pattern (meanwhile, domain size with large $m$ may also exceed the coherence length of diffraction radiation).

Now we apply this adaptive diffraction theory to the tetragonal nanotwin superlattice, as illustrated in Fig. 1(a). The twin plane in cubic notation system is (101). The twinning shear strain is $\gamma=2 \cot 2 \alpha$, where $\alpha=\tan ^{-1}\left(a_{t} / c_{t}\right)$, and $a_{t}$ and $c_{t}$ are tetragonal lattice parameters. In the coordinate system defined by $\tau$ ( $x$ axis) and $\mathbf{s}$ ( $z$ axis), the fundamental site $\mathbf{K}^{(1)}$ with index $(h k l)$ is $\mathbf{K}^{(1)}=2 \pi\left(h \cos \alpha / a_{t}\right.$ $\left.-l \sin \alpha / c_{t}, k / a_{t}, h \sin \alpha / a_{t}+l \cos \alpha / c_{t}\right)$, the twin peak splitting vector $\quad \Delta \mathbf{K}^{(1)}=-2 \pi\left(2 \cot 2 \alpha\left(h \sin \alpha / a_{t}\right.\right.$ $\left.\left.+l \cos \alpha / c_{t}\right), 0,0\right)$, the primitive superlattice translation vector $\mathbf{L}=\left[m a_{t} \cos \alpha, 0,\left(m_{1}-m_{2}\right) a_{t} \sin \alpha\right], m_{1}$ and $m_{2}$ are the numbers of (101) atomic layers in respective twin variants of the bilayer basis, $m=m_{1}+m_{2}$, the twin layer thickness $T_{1}=m_{1} a_{t} \cos \alpha, \quad T_{2}=m_{2} a_{t} \cos \alpha, \quad T=m a_{t} \cos \alpha, \quad$ and $\boldsymbol{\kappa}^{(s)}$ $=2 \pi\left(s-h m_{1}-h m_{2} \cos 2 \alpha+2 l m_{2} \sin ^{2} \alpha\right)\left(m a_{t} \cos \alpha\right)^{-1}$. Using these results in Eq. (10) determines the Bragg reflection peak intensities at $\mathbf{k}^{(s)}$.

In the following we consider the reciprocal superlattice peaks around the fundamental $(H 00)$ reflection spot (i.e., $h=H, k=l=0)$ of tetragonal $\mathrm{Pb}\left[\left(\mathrm{Mg}_{1 / 3} \mathrm{Nb}_{2 / 3}\right)_{1-x} \mathrm{Ti}_{x}\right] \mathrm{O}_{3}$ and $\mathrm{Pb}\left[\left(\mathrm{Zn}_{1 / 3} \mathrm{Nb}_{2 / 3}\right)_{1-x} \mathrm{Ti}_{x}\right] \mathrm{O}_{3}$, where the fine structure of the (200) peak has been used to identify the new monoclinic $M_{C}$ phase. $^{5-8}$ The ferroelastic strain and twin peak splitting of these materials are small, $\left(c_{t}-a_{t}\right) / a_{t} \approx \gamma / 2 \sim 0.01$, $\Delta \boldsymbol{K}^{(1)} / \boldsymbol{K}^{(1)} \sim 0.01$. While the structure factors $\tilde{n}_{1}^{0}(\mathbf{k})$ and $\tilde{n}_{2}^{0}(\mathbf{k})$ can be determined from the atomic form factors, given the pseudocubic symmetry of the crystal unit cell and the small twin peak splitting, we assume for simplicity that $\widetilde{n}_{2}^{0}\left(\mathbf{K}^{(1)}+\Delta \mathbf{K}^{(1)}\right) \approx \widetilde{n}_{1}^{0}\left(\mathbf{K}^{(1)}\right)$. The superlattice reflection peak intensity around $(H 00)$ spot is proportional to $|\Psi|^{2}$,

$$
\psi(s)=\frac{2}{\kappa_{1}} \sin \varphi_{1} e^{i \varphi_{1}}+\frac{2}{\kappa_{2}} \sin \varphi_{2} e^{-i \varphi_{2}},
$$

where $\boldsymbol{\kappa}_{1}(s)=2 \pi\left(s-H m_{1}-H m_{2} \cos 2 \alpha\right)\left(m a_{t} \cos \alpha\right)^{-1}, \boldsymbol{\kappa}_{2}(s)$ $=2 \pi\left(s-H m_{1}+H m_{1} \cos 2 \alpha\right)\left(m a_{t} \cos \alpha\right)^{-1}, \quad \varphi_{1}(s)=\pi\left(s-H m_{1}\right.$ $\left.-H m_{2} \cos 2 \alpha\right) m_{1} / m, \quad \varphi_{2}(s)=\pi\left(s-H m_{1}+H m_{1} \cos 2 \alpha\right) m_{2} / m$, and the relative phase angle is $\Delta \varphi(s)=\varphi_{1}+\varphi_{2}=\pi\left(s-H m_{1}\right)$. Using $\cos 2 \alpha \approx \gamma / 2$, it is ready to reveal that when $H m \gamma<2$, the superlattice peak $\mathbf{k}^{(s)}$ corresponding to $s_{0}$ $=H m_{1}$ has the highest intensity and is the $\mathbf{k}^{(s)}$ closest to both $\mathbf{K}^{(1)}$ and $\mathbf{K}^{(2)}$ and also the only $\mathbf{k}^{(s)}$ located between them. ${ }^{12}$ For the (200) spot, the condition $H m \gamma<2$ gives $m<50$, i.e., the bilayer basis is thinner than $14 \mathrm{~nm}$ (with $a_{t} \approx 0.4 \mathrm{~nm}$ ). The superlattice reflection peak corresponding to $s_{0}=\mathrm{Hm}_{1}$ is the one that is observed in experiments (for example, when $m_{1}=m_{2}=10$, the superlattice peaks corresponding to $s=s_{0} \pm 1$ and \pm 2 have respective intensities of 0.0163 and 0.0001 with respect to that of $s=s_{0}$ ). The position of this observed superlattice peak is $\mathbf{k}^{(s)}=\mathbf{K}^{(1)}+(1-\omega) \Delta \mathbf{K}^{(1)}$, as illustrated in Fig. 1(b), where $\omega=m_{1} / m$ is the twin variant volume fraction. Therefore, this superlattice reflection peak $\mathbf{k}^{(s)}$ adaptively shifts between $\mathbf{K}^{(1)}$ and $\mathbf{K}^{(2)}$ along the twin peak splitting vector $\Delta \mathbf{K}^{(1)}$ according to the twin variant volume fraction $\omega$. The lattice parameter measured from this $\mathbf{k}^{(s)}$ around the $(H 00)$ spot is $a \approx \omega a_{t}+(1-\omega) c_{t}$. The same adaptive behavior also occurs around the $(00 L)$ spot, which gives a lattice parameter $c \approx \omega c_{t}+(1-\omega) a_{t}$. The lattice parameter measured from the $(0 K 0)$ spot is $b=a_{t}$, because the nanotwin superlattice has homogeneous $\mathbf{b}_{1}=\mathbf{b}_{2}$ lattice translation in the $y$-axis direction, and the $(0 K 0)$ peak does not exhibit such an adaptive reflection behavior. It is noted that these lattice parameter relationships are the same as obtained from the crystallographic analysis of tetragonal twins by volume fraction averaging. ${ }^{9,10}$ Therefore, the adaptive diffraction theory validates the previously used twin-domain averaging procedure. ${ }^{9,10}$ It is also noted that each set of tetragonal nanotwin superlattice diffracts incident waves just like one monoclinic $M_{C}$ domain. When multiple sets of nanotwins of different $\{101\}$ twin planes are present, the effect is just the same as the presence of multiple $M_{C}$ domains of different 


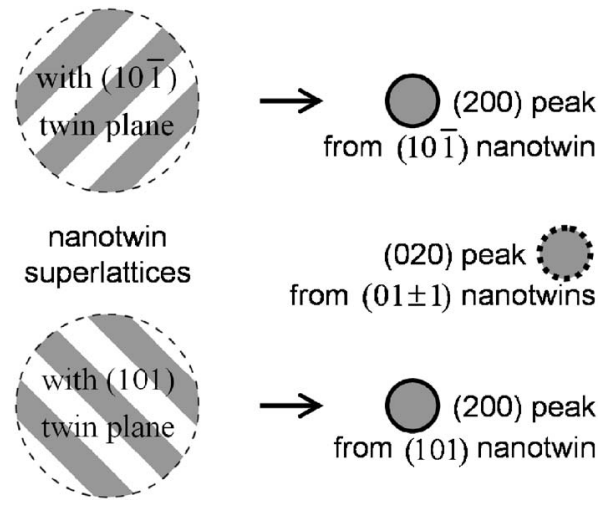

FIG. 2. Triplet peak structure around the (200) Bragg reflection spot consisting of two (200) superlattice peaks from nanotwins of $(10 \overline{1})$ and (101) twin planes, respectively, and one (020) superlattice peak from nanotwins of $(01 \pm 1)$ twin planes. The vanished fundamental peaks are not shown. Nanotwins of $(01 \pm 1)$ twin planes (not shown) produce the same (020) peak (highlighted by dotted line) as that from [001]-oriented tetragonal domain and give the lattice parameter $b=a_{t}$.

orientations, which gives rise to the observed characteristic triplet structure of the $(H 00)$ reflection peak, ${ }^{5-8}$ which has led to an interpretation of $M_{C}$ phase. As illustrated in Fig. 2, the triplet peak structure around the (200) Bragg reflection spot consists of two (200) superlattice peaks from nanotwins of $(10 \overline{1})$ and (101) twin planes, respectively, and one (020) superlattice peak from nanotwins of $(011)$ and/or $(01 \overline{1})$ twin planes. The (200) superlattice peaks give the lattice parameter $a \approx \omega a_{t}+(1-\omega) c_{t}$. The nanotwins of (011) and $(01 \overline{1})$ twin planes (not shown in Fig. 2) produce the same (020) peak (highlighted by dotted line) as that produced by [001]oriented tetragonal domain and give the lattice parameter $b=a_{t}$. This analysis also explains the superlattice peaks observed in powder diffraction. ${ }^{13-16}$ Recently, nanotwins of tetragonal phase with domain size about $10 \mathrm{~nm}$ has been directly observed by transmission electron microscopy (TEM) in $\mathrm{Pb}\left[\left(\mathrm{Mg}_{1 / 3} \mathrm{Nb}_{2 / 3}\right)_{1-x} \mathrm{Ti}_{x}\right] \mathrm{O}_{3} \cdot{ }^{17}$

It is worth noting that deviations from a perfect nanotwin superlattice through fluctuations in $m_{1}$ and $m_{2}$, such as variations in the bilayer basis thickness and twin volume fraction, will produce peak broadening. As in our ongoing research, such an effect is under investigation by using a computational approach to explicitly account for the imperfections in nanotwin superlattices. Nevertheless, the calculation of perfect nanotwin superlattices presented in this paper predicts the lattice parameters and their intrinsic relationships, which are in agreement with the diffraction experiments. ${ }^{5-8,13-16}$

In summary, we develop a diffraction theory of nanotwin superlattices of low symmetry phases. This theory predicts an adaptive diffraction phenomenon, where the Bragg reflection peaks are located at reciprocal superlattice sites in the immediate vicinity of fundamental spots of the constituent crystals and adaptively shift along the twin peak splitting vectors in response to a change in twin variant volume fraction. We apply this theory to tetragonal nanotwin superlattice and show that diffraction perceives tetragonal nanotwins as monoclinic $M_{C}$ phase, whose lattice parameters are intrinsically related to that of the tetragonal phase. It explains the recent experimental observations in $\mathrm{Pb}\left[\left(\mathrm{Mg}_{1 / 3} \mathrm{Nb}_{2 / 3}\right)_{1-x} \mathrm{Ti}_{x}\right] \mathrm{O}_{3}$ and $\mathrm{Pb}\left[\left(\mathrm{Zn}_{1 / 3} \mathrm{Nb}_{2 / 3}\right)_{1-x} \mathrm{Ti}_{x}\right] \mathrm{O}_{3}$. Similar adaptive diffraction phenomena should occur in other nanoscale superlattices of low symmetry phases.

\section{ACKNOWLEDGMENT}

Financial support from Virginia Tech through a startup fund is acknowledged.
*Email: yuwang@mse.vt.edu

${ }^{1}$ A. G. Khachaturyan, Theory of Structural Transformations in Solids (John Wiley \& Sons, New York, 1983).

${ }^{2}$ A. L. Roytburd, J. Phys. IV, C8 21 (1995).

${ }^{3}$ A. G. Khachaturyan, S. M. Shapiro, and S. Semenovskaya, Phys. Rev. B 43, 10832 (1991).

${ }^{4}$ S. M. Shapiro, B. X. Yang, G. Shirane, Y. Noda, and L. E. Tanner, Phys. Rev. Lett. 62, 1298 (1989).

${ }^{5}$ B. Noheda, D. E. Cox, G. Shirane, S. E. Park, L. E. Cross, and Z. Zhong, Phys. Rev. Lett. 86, 3891 (2001).

${ }^{6}$ B. Noheda, Z. Zhong, D. E. Cox, G. Shirane, S. E. Park, and P. Rehrig, Phys. Rev. B 65, 224101 (2002).

${ }^{7}$ K. Ohwada, K. Hirota, P. W. Rehrig, Y. Fujii, and G. Shirane, Phys. Rev. B 67, 094111 (2003).

${ }^{8}$ F. Bai, N. Wang, J. Li, D. Viehland, P. M. Gehring, G. Xu, and G. Shirane, J. Appl. Phys. 96, 1620 (2004).

${ }^{9}$ Y. M. Jin, Y. U. Wang, A. G. Khachaturyan, J. F. Li, and D. Viehland, Phys. Rev. Lett. 91, 197601 (2003); J. Appl. Phys. 94, 3629 (2003).
${ }^{10}$ Y. U. Wang, Phys. Rev. B 73, 014113 (2006).

${ }^{11}$ Y. M. Jin and G. J. Weng, Acta Mater. 50, 2967 (2002).

${ }^{12}$ In fact, $s_{0}=H m_{1}$ gives the largest magnitudes for both terms in Eq. (12) with constructive superposition $(\Delta \varphi=0)$, while the next superlattice peaks correspond to $s=s_{0} \pm 1$ with $\Delta \varphi= \pm \pi$ (destructive superposition) and are located outside of the twin peak splitting vector $\Delta \mathbf{K}^{(1)}$, thus having very small intensities. The peaks further away have almost vanishing intensities because of rapidly decreased magnitudes of both terms in Eq. (12).

${ }^{13}$ D. E. Cox, B. Noheda, G. Shirane, Y. Uesu, K. Fujishiro, and Y. Yamada, Appl. Phys. Lett. 79, 400 (2001).

${ }^{14}$ D. La-Orauttapong, B. Noheda, Z. G. Ye, P. M. Gehring, J. Toulouse, D. E. Cox, and G. Shirane, Phys. Rev. B 65, 144101 (2002).

${ }^{15}$ B. Noheda, D. E. Cox, G. Shirane, J. Gao, and Z. G. Ye, Phys. Rev. B 66, 054104 (2002).

${ }^{16}$ A. K. Singh and D. Pandey, Phys. Rev. B 67, 064102 (2003).

${ }^{17}$ H. Wang, J. Zhu, N. Lu, A. A. Bokov, Z. G. Ye, and X. W. Zhang, Appl. Phys. Lett. 89, 042908 (2006). 